\title{
Valuation of
}

\section{American Continuous-Installment Options *}

\author{
P. Ciurlia \\ Dipartimento di Metodi Quantitativi \\ Università degli Studi di Brescia \\ ciurlia@eco.unibs.it \\ I. Roko \\ Department of Econometrics \\ University of Geneva \\ ilir.roko@metri.unige.ch
}

October 2004

\begin{abstract}
We present three approaches to value American continuous-installment calls and puts and compare their computational precision. In an American continuous-installment option, the premium is paid continuously instead of up-front. At or before maturity, the holder may terminate payments by either exercising the option or stopping the option contract. Under the usual assumptions, we are able to construct an instantaneous riskless dynamic hedging portfolio and derive an inhomogeneous Black-Scholes partial differential equation for the initial value of this option. This key result allows us to derive valuation formulas for American continuous-installment options using the integral representation method and consequently to obtain closed-form formulas by approximating the optimal stopping and exercise boundaries as multipiece exponen-
\end{abstract}

*We are grateful to Manfred Gilli, Henri Loubergé and Evis Këllezi for encouragements, suggestions and remarks. 
tial functions. This process is compared to the finite-difference method to solve the inhomogeneous Black-Scholes PDE and a Monte Carlo approach.

\section{Introduction}

In a conventional American-style option contract, the buyer pays the premium entirely upfront and acquires the right, but not the obligation, to exercise the option at any time up to a fixed maturity time $T$. Here we consider an alternative form of American-style option contract in which the buyer pays a smaller up-front premium and then a constant stream of installments at a certain rate per unit time. However, the buyer can choose at any time to stop making installment payments either by exercising the option or by stopping the option contract.

There is little literature on installment options. Davis et al. (2001, 2002) derive noarbitrage bounds for the initial premium of a discretely-paid installment option and study static versus dynamic hedging strategies within a Black-Scholes framework with stochastic volatility. Their analysis is restricted to European-style installment options, which allows for an analogy with compound options, previously considered in Geske (1977) and Selby and Hodges (1987). Davis et al. (2003) values venture capital using an analogy with the installment option. Ben-Ameur et al. (2004) develops a dynamic-programming procedure to price American-style installment options and derive some theoretical properties of the installment option contract within the geometric Brownian motion framework. Their approach is applied to installment warrants, which are actively traded on the Australian Stock Exchange. Finally, Wystup et al. (2004) compares pricing techniques for installment options written on exchange rates.

The aim of this paper is to present three alternative approaches for valuing American continuous-installment calls and puts and to compare their computational advantages. In Section 2, we formulate the American continuous-installments option valuation problem as a free boundary-value problem and obtain an analytic solution by utilizing the results in Carr et al. (1992), Jacka (1991) and Kim (1990) ${ }^{1}$. In Section 3, we describe in detail the alternative approaches. Numerical results are compared in Section 4. Section 5 concludes.

\footnotetext{
${ }^{1}$ We are grateful to Steward Hodges for suggesting this approach.
} 


\section{American Continuous-Installment Options}

The particular feature of the pricing problem of an American continuous-installment option is the determination, along with the initial premium and the optimal exercise boundary, of a further boundary called the optimal stopping boundary.

\subsection{Black-Scholes PDE for Continuous-Installment Options}

We assume the standard model for perfect capital markets, continuous trading, no-arbitrage opportunities, a constant interest rate $r>0$, and an asset paying continuous proportional dividends $\delta>0$ with price $S_{t}$ following a geometric Brownian motion

$$
d S_{t}=\mu S_{t} d t+\sigma S_{t} d B_{t}
$$

where $\mu=(r-\delta)$ and $d B_{t}$ is a Wiener process on a risk-neutral probability space. The Black-Scholes initial premium $V$ of a continuous-installment option

$$
V_{t}=V\left(S_{t}, t ; q\right)
$$

depends on the current value of the underlying asset $S_{t}$, time $t$, and the continuous installment rate $q$. Applying Itô's Lemma to (2) we obtain the dynamics for the initial value of this option

$$
d V_{t}=\left(\frac{\partial V_{t}}{\partial t}+\mu S_{t} \frac{\partial V_{t}}{\partial S}+\frac{1}{2} \sigma^{2} S_{t}^{2} \frac{\partial^{2} V_{t}}{\partial S^{2}}-q\right) d t+\sigma S_{t} \frac{\partial V_{t}}{\partial S} d B_{t}
$$

The only difference in expression (3) relative to the standard Black-Scholes framework is the presence of the constant rate $q$ that has to be paid to stay in the option contract.

We now construct the replicating portfolio consisting of one continuous-installment option and an amount $-\Phi$ of the underlying asset. The value of this portfolio is

$$
\Pi_{t}=V_{t}-\Phi S_{t}
$$

and its dynamics is given by

$$
d \Pi_{t}=d V_{t}-\Phi d S_{t}-\Phi\left(S_{t} \delta d t\right)
$$


Putting (1) and (3) together, we get

$$
d \Pi_{t}=\left(\mu S_{t}\left(\frac{\partial V_{t}}{\partial S}-\Phi\right)+\frac{\partial V_{t}}{\partial t}+\frac{1}{2} \sigma^{2} S_{t}^{2} \frac{\partial^{2} V_{t}}{\partial S^{2}}-q-\Phi S_{t} \delta\right) d t+\sigma S_{t}\left(\frac{\partial V_{t}}{\partial S}-\Phi\right) d B_{t}
$$

Setting $\Phi=\frac{\partial V_{t}}{\partial S}$ the coefficient of $d B_{t}$ vanishes. The portfolio is instantaneously riskless and, to avoid arbitrage opportunities, must yield return $r$. So we must have

$$
r\left(V_{t}-\frac{\partial V_{t}}{\partial S} S_{t}\right)=\left(\frac{\partial V_{t}}{\partial t}+\frac{1}{2} \sigma^{2} S_{t}^{2} \frac{\partial^{2} V_{t}}{\partial S^{2}}-q-\frac{\partial V_{t}}{\partial S} S_{t} \delta\right)
$$

Rearranging this equation gives the inhomogeneous Black-Scholes PDE for the initial premium of a continuous-installment option

$$
\frac{\partial V_{t}}{\partial t}+\mu S_{t} \frac{\partial V_{t}}{\partial S}+\frac{1}{2} \sigma^{2} S_{t}^{2} \frac{\partial^{2} V_{t}}{\partial S^{2}}-r V_{t}=q
$$

\subsection{Valuation of American Continuous-Installment Calls}

Consider an American continuous-installment call on $S_{t}$ with strike price $K$ and maturity time $T$. We denote the initial premium of this call at time $t$ by $C\left(S_{t}, t ; q\right)$, defined on the domain $\mathcal{D}=\left\{\left(S_{t}, t\right) \in[0, \infty[\times[0, T]\}\right.$. For each time $t \in[0, T]$, there exists an upper critical asset price $B_{t}$ above which it is optimal to stop the installment payments by exercising the option early, as well as a lower critical asset price $A_{t}$ below which it is advantageous to terminate payments by stopping the option contract. According to these upper and lower critical asset prices the initial premium $C\left(S_{t}, t ; q\right)$ is

$$
\begin{array}{ll}
C\left(S_{t}, t ; q\right)=\left(S_{t}-K\right)_{+} & \text {if } S_{t} \in\left[0, A_{t}\right] \cup\left[B_{t}, \infty[\right. \\
C\left(S_{t}, t ; q\right)>\left(S_{t}-K\right)_{+} & \text {if } \left.S_{t} \in\right] A_{t}, B_{t}[
\end{array}
$$

The stopping and exercise boundaries are the time paths of lower and upper critical asset prices $A_{t}$ and $B_{t}$, for $t \in[0, T]$, respectively. These boundaries divide the domain $\mathcal{D}$ into a stopping region $\mathcal{D}_{1}=\left\{\left(S_{t}, t\right) \in\left[0, A_{t}\right] \times[0, T]\right\}$, a continuation region $\mathcal{D}_{2}=\left\{\left(S_{t}, t\right) \in\right.$ ]$A_{t}, B_{t}[\times[0, T]\}$, and an exercise region $\mathcal{D}_{3}=\left\{\left(S_{t}, t\right) \in\left[B_{t}, \infty[\times[0, T]\}\right.\right.$.

To ensure that the fundamental constraint $C\left(S_{t}, t ; q\right) \geq\left(S_{t}-K\right)_{+}$is satisfied in the domain $\mathcal{D}$, equation (5) impose that, in the stopping and exercise regions, the initial premium 
$C\left(S_{t}, t ; q\right)$ equals to the option payoff $\left(S_{t}-K\right)_{+}$. By contrast, the inequality expressed in (6) shows that, in the continuation region, it is advantageous to continue paying the installment premiums since the call is worth more alive than dead. The initial premium is given by (5) if the asset price starts either in $\mathcal{D}_{1}$ or $\mathcal{D}_{3}$, so we assume that the call is alive at the valuation time 0, i.e., $A_{0}<S_{0}<B_{0}$.

The initial premium $C\left(S_{t}, t ; q\right)$ of the American continuous-installment call satisfies the inhomogeneous Black-Scholes PDE (4) in $\mathcal{D}_{2}$; that is,

$$
\frac{\partial C\left(S_{t}, t ; q\right)}{\partial t}+\mu S_{t} \frac{\partial C\left(S_{t}, t ; q\right)}{\partial S}+\frac{1}{2} \sigma^{2} S_{t}^{2} \frac{\partial^{2} C\left(S_{t}, t ; q\right)}{\partial S^{2}}-r C\left(S_{t}, t ; q\right)=q \quad \text { on } \mathcal{D}_{2} .
$$

Extending the analysis of McKean (1965), we determine that $C\left(S_{t}, t ; q\right)$ and the stopping and exercise boundaries $A_{t}$ and $B_{t}$ jointly solve a free boundary-value problem consisting of (7) subject to the following final and boundary conditions:

$$
\begin{aligned}
& C\left(S_{T}, T ; q\right)=\left(S_{T}-K\right)_{+} \\
& \lim _{S_{t} \downarrow A_{t}} C\left(S_{t}, t ; q\right)=0 \\
& \lim _{S_{t} \downarrow A_{t}} \frac{\partial C\left(S_{t}, t ; q\right)}{\partial S}=0 \\
& \lim _{S_{t} \uparrow B_{t}} C\left(S_{t}, t ; q\right)=B_{t}-K \\
& \lim _{S_{t} \uparrow B_{t}} \frac{\partial C\left(S_{t}, t ; q\right)}{\partial S}=1 .
\end{aligned}
$$

The value matching conditions (9) and (11) imply that the initial premium is continuous across the stopping and exercise boundaries, respectively. Furthermore, the high contact conditions (10) and (12) further imply that the slope is continuous. Equations (9-12) are jointly referred to as smooth fit conditions and ensure the optimality of the stopping and exercise boundaries.

We solve this problem with the integral representation method introduced in Carr et al. (1992), Jacka (1991) and Kim (1990). Ziogas et al. (2004) presents a survey of the methods for deriving the various integral representations of American option prices.

Let $Z\left(S_{t}, t\right) \equiv e^{-r t} C\left(S_{t}, t ; q\right)$ be the discounted initial premium function of the American continuous-installment call, defined in the domain $\mathcal{D}$. In this domain, the function $Z\left(S_{t}, t\right)$ inherits the properties of the initial premium function $C\left(S_{t}, t ; q\right)$, i.e., it is a convex 
function in $S_{t}$ for all $t$, continuously differentiable in $t$ for all $S_{t}$ and a.e. twice continuously differentiable in $S_{t}$ for all $t$. Applying Itô's Lemma to $Z\left(S_{t}, t\right)$ yields

$$
Z\left(S_{T}, T\right)=Z\left(S_{0}, 0\right)+\int_{0}^{T} \frac{\partial Z\left(S_{t}, t\right)}{\partial S} d S_{t}+\int_{0}^{T}\left(\frac{\sigma^{2}}{2} S_{t}^{2} \frac{\partial^{2} Z\left(S_{t}, t\right)}{\partial S^{2}}+\frac{\partial Z\left(S_{t}, t\right)}{\partial t}\right) d t
$$

In terms of $C\left(S_{t}, t ; q\right)$ this means

$$
\begin{aligned}
e^{-r T} C\left(S_{T}, T ; q\right)= & C\left(S_{0}, 0 ; q\right)+\int_{0}^{T} e^{-r t} \frac{\partial C\left(S_{t}, t ; q\right)}{\partial S} d S_{t} \\
& +\int_{0}^{T} e^{-r t}\left(\frac{\sigma^{2}}{2} S_{t}^{2} \frac{\partial^{2} C\left(S_{t}, t ; q\right)}{\partial S^{2}}-r C\left(S_{t}, t ; q\right)+\frac{\partial C\left(S_{t}, t ; q\right)}{\partial t}\right) d t
\end{aligned}
$$

From (8) we know that $C\left(S_{T}, T ; q\right)=\left(S_{T}-K\right)_{+}$and, separating the initial premium into $C\left(S_{t}, t ; q\right)=\mathbf{1}_{\left\{A_{t}<S_{t}<B_{t}\right\}} C\left(S_{t}, t ; q\right)+\mathbf{1}_{\left\{S_{t} \geq B_{t}\right\}}\left(S_{t}-K\right)$, we have

$$
\begin{aligned}
e^{-r T}\left(S_{T}-K\right)_{+}= & C\left(S_{0}, 0 ; q\right) \\
& +\int_{0}^{T} e^{-r t}\left(\mathbf{1}_{\left\{A_{t}<S_{t}<B_{t}\right\}} \frac{\partial C\left(S_{t}, t ; q\right)}{\partial S}+\mathbf{1}_{\left\{S_{t} \geq B_{t}\right\}}\right)\left(\mu S_{t} d t+\sigma S_{t} d B_{t}\right) \\
& +\int_{0}^{T} e^{-r t} \mathbf{1}_{\left\{A_{t}<S_{t}<B_{t}\right\}}\left(\frac{\sigma^{2}}{2} S_{t}^{2} \frac{\partial^{2} C\left(S_{t}, t ; q\right)}{\partial S^{2}}-r C\left(S_{t}, t ; q\right)+\frac{\partial C\left(S_{t}, t ; q\right)}{\partial t}\right) d t \\
& +\int_{0}^{T} e^{-r t} \mathbf{1}_{\left\{S_{t} \geq B_{t}\right\}}\left(-r\left(S_{t}-K\right)\right) d t .
\end{aligned}
$$

On the continuation region, the initial premium function $C\left(S_{t}, t ; q\right)$ satisfies the inhomogeneous Black-Scholes PDE (7), so the terms multiplying $\mathbf{1}_{\left\{A_{t}<S_{t}<B_{t}\right\}}$ sum to $q$. Using this, and taking expectations, reduces the above equation to

$$
\begin{aligned}
c_{0} \equiv E\left[e^{-r T}\left(S_{T}-K\right)_{+}\right]= & C\left(S_{0}, 0 ; q\right)+q \int_{0}^{T} e^{-r t}\left(N\left(d_{2}\left(S_{0}, A_{t}, t\right)\right)-N\left(d_{2}\left(S_{0}, B_{t}, t\right)\right)\right) d t \\
& +\int_{0}^{T} e^{-r t}\left(-\delta S_{0} e^{(r-\delta) t} N\left(d_{1}\left(S_{0}, B_{t}, t\right)\right)+r K N\left(d_{2}\left(S_{0}, B_{t}, t\right)\right)\right) d t .
\end{aligned}
$$

By rearranging this expression, we obtain the integral representation for the initial premium of the American continuous-installment call:

$$
\begin{aligned}
C\left(S_{0}, 0 ; q\right)= & c_{0}+\int_{0}^{T}\left(\delta S_{0} e^{-\delta t} N\left(d_{1}\left(S_{0}, B_{t}, t\right)\right)+(q-r K) e^{-r t} N\left(d_{2}\left(S_{0}, B_{t}, t\right)\right)\right) d t \\
& -q \int_{0}^{T} e^{-r t} N\left(d_{2}\left(S_{0}, A_{t}, t\right)\right) d t
\end{aligned}
$$


where

$$
d_{1}(x, y, t)=\frac{\ln (x / y)+\left(r-\delta+\sigma^{2} / 2\right) t}{\sigma \sqrt{t}} \quad \text { and } \quad d_{2}(x, y, t)=d_{1}(x, y, t)-\sigma \sqrt{t}
$$

and $c_{0}$ is the Black-Scholes/Merton European call pricing formula.

Equation (13) expresses the initial premium of an American continuous-installment call as the sum of the corresponding European call value, the early exercise premium, and the expected present value of installment payments along the optimal stopping boundary. The early exercise premium can be viewed as the value of a contingent claim that allows interest earned on the strike price, decreased by the installment premium, to be changed for dividends paid by the asset whenever the asset price is above the optimal exercise boundary.

The optimal stopping boundary $A_{t}$ is implicitly defined by the following integral equation:

$$
\begin{aligned}
0= & c_{t}\left(A_{t}, K, T-t\right) \\
& +\int_{t}^{T}\left(\delta A_{t} e^{-\delta(s-t)} N\left(d_{1}\left(A_{t}, B_{s}, s-t\right)\right)+(q-r K) e^{-r(s-t)} N\left(d_{2}\left(A_{t}, B_{s}, s-t\right)\right)\right) d s \\
& -q \int_{t}^{T} e^{-r(s-t)} N\left(d_{2}\left(A_{t}, A_{s}, s-t\right)\right) d s .
\end{aligned}
$$

Equation (14) reflects the fact that the initial premium of an American continuous-installment call at the time of optimal stopping is equal to the option payoff, which is zero. Similarly, applying the boundary condition (11), we obtain the integral equation satisfied by the optimal exercise boundary $B_{t}$ :

$$
\begin{aligned}
B_{t}-K= & c_{t}\left(B_{t}, K, T-t\right) \\
& +\int_{t}^{T}\left(\delta B_{t} e^{-\delta(s-t)} N\left(d_{1}\left(B_{t}, B_{s}, s-t\right)\right)+(q-r K) e^{-r(s-t)} N\left(d_{2}\left(B_{t}, B_{s}, s-t\right)\right)\right) d s \\
& -q \int_{t}^{T} e^{-r(s-t)} N\left(d_{2}\left(B_{t}, A_{s}, s-t\right)\right) d s .
\end{aligned}
$$

This suggests that the initial premium of American continuous-installment calls should be computed in two steps. In the first, (14) and (15) are solved for $A_{t}$ and $B_{t}$, respectively. Given the optimal stopping and exercise boundaries, (13) is solved next. Unfortunately, direct solutions for the integral equations (14) and (15) are not possible. According to Kolodner (1956), these are Volterra integral equations and can only be solved numerically. 
In Section 3.1, we present a numerical approximation method for solving (13) directly in closed form.

\subsection{Valuation of American Continuous-Installment Puts}

For the valuation of an American continuous-installment put we proceed in the same way as for the call. We denote by $P\left(S_{t}, t ; q\right)$, defined on the same domain $\mathcal{D}$, the initial premium function of the American continuous-installment put.

For each time $t$, there must be a lower critical asset price $F_{t}$ below which it is optimal to terminate payments by exercising the option, as well as an upper critical asset price $G_{t}$ above which it is advantageous to terminate payments by stopping the option contract. The exercise and stopping boundaries, which are the time paths of lower and upper critical asset prices $F_{t}$ and $G_{t}$, divide the domain $\mathcal{D}$ into an exercise region $\widetilde{\mathcal{D}}_{1}=\left\{\left(S_{t}, t\right) \in\left[0, F_{t}\right] \times[0, T]\right\}$, a continuation region $\widetilde{\mathcal{D}}_{2}=\left\{\left(S_{t}, t\right) \in\right] F_{t}, G_{t}[\times[0, T]\}$, and a stopping region $\widetilde{\mathcal{D}}_{3}=\left\{\left(S_{t}, t\right) \in\right.$ $\left[G_{t}, \infty[\times[0, T]\}\right.$.

The initial premium function $P\left(S_{t}, t ; q\right)$ satisfies the inhomogeneous Black-Scholes PDE in the continuation region $\widetilde{\mathcal{D}}_{2}$; that is,

$$
\frac{\partial P\left(S_{t}, t ; q\right)}{\partial t}+\mu S_{t} \frac{\partial P\left(S_{t}, t ; q\right)}{\partial S}+\frac{1}{2} \sigma^{2} S_{t}^{2} \frac{\partial^{2} P\left(S_{t}, t ; q\right)}{\partial S^{2}}-r P\left(S_{t}, t ; q\right)=q \quad \text { on } \widetilde{\mathcal{D}}_{2}
$$

subject to the following terminal and boundary conditions

$$
\begin{aligned}
& P\left(S_{T}, T ; q\right)=\left(K-S_{T}\right)_{+} \\
& \lim _{S_{t} \downarrow F_{t}} P\left(S_{t}, t ; q\right)=K-F_{t} \\
& \lim _{S_{t} \downarrow F_{t}} \frac{\partial P\left(S_{t}, t ; q\right)}{\partial S}=-1 \\
& \lim _{S_{t} \uparrow G_{t}} P\left(S_{t}, t ; q\right)=0 \\
& \lim _{S_{t} \uparrow G_{t}} \frac{\partial P\left(S_{t}, t ; q\right)}{\partial S}=0 .
\end{aligned}
$$

By applying the results of the previous section, the solution to the free boundary-value 
problem $(16-21)$ is

$$
\begin{aligned}
P\left(S_{0}, 0 ; q\right)= & p_{0}+\int_{0}^{T}\left((q+r K) e^{-r t} N\left(-d_{2}\left(S_{0}, F_{t}, t\right)\right)-S_{0} \delta e^{-\delta t} N\left(-d_{1}\left(S_{0}, F_{t}, t\right)\right)\right) d t \\
& -q \int_{0}^{T} e^{-r t} N\left(-d_{2}\left(S_{0}, G_{t}, t\right)\right) d t
\end{aligned}
$$

Using the property of the normal cdf, we can rewrite the equation (22) as

$$
\begin{aligned}
P\left(S_{0}, 0 ; q\right)= & p_{0}+\frac{1}{r}(q+r K)\left(1-e^{-r T}\right)-S_{0}\left(1-e^{-\delta T}\right) \\
& +\int_{0}^{T}\left(S_{0} \delta e^{-\delta t} N\left(d_{1}\left(S_{0}, F_{t}, t\right)\right)-(q+r K) e^{-r t} N\left(d_{2}\left(S_{0}, F_{t}, t\right)\right)\right) d t \\
& -\frac{q}{r}\left(1-e^{-r T}\right)+q \int_{0}^{T} e^{-r t} N\left(d_{2}\left(S_{0}, G_{t}, t\right)\right) d t
\end{aligned}
$$

Applying the boundary conditions (18) and (20), we obtain the integral equations for $F_{t}$ and $G_{t}$ :

$$
\begin{aligned}
\left(K-F_{t}\right)= & p_{t}\left(F_{t}, K, T-t\right)+\frac{1}{r}(q+r K)\left(1-e^{-r(T-t)}\right)-F_{t}\left(1-e^{-\delta(T-t)}\right) \\
& +\int_{t}^{T}\left(F_{t} \delta e^{-\delta(s-t)} N\left(d_{1}\left(F_{t}, F_{s}, s-t\right)\right)-(q+r K) e^{-r(s-t)} N\left(d_{2}\left(F_{t}, F_{s}, s-t\right)\right)\right) d s \\
& \quad-\frac{q}{r}\left(1-e^{-r(T-t)}\right)+q \int_{t}^{T} e^{-r(s-t)} N\left(d_{2}\left(F_{t}, G_{s}, s-t\right)\right) d s \\
0= & p_{t}\left(G_{t}, K, T-t\right)+\frac{1}{r}(q+r K)\left(1-e^{-r(T-t)}\right)-G_{t}\left(1-e^{-\delta(T-t)}\right) \\
& +\int_{t}^{T}\left(G_{t} \delta e^{-\delta(s-t)} N\left(d_{1}\left(G_{t}, F_{s}, s-t\right)\right)-(q+r K) e^{-r(s-t)} N\left(d_{2}\left(G_{t}, F_{s}, s-t\right)\right)\right) d s \\
& -\frac{q}{r}\left(1-e^{-r(T-t)}\right)+q \int_{t}^{T} e^{-r(s-t)} N\left(d_{2}\left(G_{t}, G_{s}, s-t\right)\right) d s .
\end{aligned}
$$

\section{$3 \quad$ Numerical Methods}

Here we present the three alternative approaches to value the American continuous-installment options. First we implement the valuation formulas derived in Section 2 using the multipiece exponential function method of Ju (1998). Second the finite-difference method for solving the inhomogeneous Black-Scholes PDE is presented. Finally, we consider a Monte Carlo method. 


\subsection{Implementation of the Valuation Formulas by the Multipiece Exponential Function (MEF) Method}

Once the integral equations defining the optimal stopping and exercise boundaries are solved, the computation of the initial premium simply implies numerical integration. Unfortunately, these integral equations cannot be solved explicitly. However, there is a special feature of equations (13) and (23) that has been investigated in the literature. Noting that the exercise boundary appears only as an argument to the logarithm function in the definitions of $d_{1}(\cdot)$ and $d_{2}(\cdot)$, Ju (1998) argues that the integral equation for the American put value does not depend on the exact values of the exercise boundary critically. Making use of this property and approximating the boundary as a multipiece exponential function, he obtains a closed-form formula for pricing American-style options.

To extend the approach in Ju (1998), hereafter called the Multipiece Exponential Function (MEF) method, we divide the interval $[0, T]$ into $M$ equal time intervals and define $t_{j}=j T / M, j=1,2, \ldots, M$. Let $C^{\mathrm{CI}}$ be the approximated initial premium of an American continuous-installment call corresponding to the approximated optimal stopping and exercise boundaries by $M$-piece exponential functions $A_{j} e^{a_{j} t}$ and $B_{j} e^{-b_{j} t}$, for $j=1,2, \ldots, M$, respectively. Then $C^{\mathrm{CI}}$ is given by

$$
C^{\mathrm{CI}}= \begin{cases}0 & \text { if } \quad S_{0} \leq A_{M} \\ C\left(M, S_{0}, A, B, a, b, \phi, \nu, T\right) & \text { if } \quad A_{M}<S_{0}<B_{M} \\ S_{0}-K & \text { if } \quad S_{0} \geq B_{M},\end{cases}
$$

where

$$
\begin{aligned}
C(j, x, A, B, a, b, \phi, \nu, \tau)= & c(x, K, \tau)-q \sum_{i=1}^{j} I\left(t_{i-1}, t_{i}, x, A_{j-i+1} e^{a_{j-i+1}(T-\tau)}, a_{j-i+1},-1, r\right) \\
+ & \left(x \sum_{i=1}^{j} \delta I\left(t_{i-1}, t_{i}, x, B_{j-i+1} e^{-b_{j-i+1}(T-\tau)},-b_{j-i+1}, 1, \delta\right)\right. \\
& \left.+(q-r K) \sum_{i=1}^{j} I\left(t_{i-1}, t_{i}, x, B_{j-1+1} e^{-b_{j-i+1}(T-\tau)},-b_{j-i+1},-1, r\right)\right) .
\end{aligned}
$$

To determine the coefficients $A_{j}, a_{j}, B_{j}$ and $b_{j}, j=1,2, \ldots, M$, we apply the value-match 
and high-contact conditions (9-12) at each time step $t_{j}$. This yields

$$
\left\{\begin{array}{l}
C\left(j, A_{j} e^{a_{j}\left(T-t_{j}\right)}, A, B, a, b, \phi, \nu, t_{j}\right)=0 \\
C_{x}\left(j, A_{j} e^{a_{j}\left(T-t_{j}\right)}, A, B, a, b, \phi, \nu, t_{j}\right)=0 \\
C\left(j, B_{j} e^{-b_{j}\left(T-t_{j}\right)}, A, B, a, b, \phi, \nu, t_{j}\right)=B_{j} e^{-b_{j}\left(T-t_{j}\right)}-K \\
C_{x}\left(j, B_{j} e^{-b_{j}\left(T-t_{j}\right)}, A, B, a, b, \phi, \nu, t_{j}\right)=1
\end{array}\right.
$$

where

$$
\begin{aligned}
C_{x}(j, x, A, B, a, b, \phi, \nu, \tau)= & e^{-\delta \tau} N\left(d_{1}(x, K, \tau)\right) \\
& -q \sum_{i=1}^{j} I_{x}\left(t_{i-1}, t_{i}, x, A_{j-i+1} e^{a_{j-i+1}(T-\tau)}, a_{j-i+1},-1, r\right) \\
& +\sum_{i=1}^{j} \delta I\left(t_{i-1}, t_{i}, x, B_{j-i+1} e^{-b_{j-i+1}(T-\tau)},-b_{j-i+1}, 1, \delta\right) \\
& +x \sum_{i=1}^{j} \delta I_{x}\left(t_{i-1}, t_{i}, x, B_{j-i+1} e^{-b_{j-i+1}(T-\tau)},-b_{j-i+1}, 1, \delta\right) \\
& +(q-r K) \sum_{i=1}^{j} I_{x}\left(t_{i-1}, t_{i}, x, B_{j-i+1} e^{-b_{j-i+1}(T-\tau)},-b_{j-i+1},-1, r\right) .
\end{aligned}
$$

The functions $I(\cdot)$ and $I_{x}(\cdot)$ are defined, respectively, by ${ }^{2}$

$$
\begin{gathered}
I\left(t_{i-1}, t_{i}, x, y, z, \phi, \nu\right)=\frac{1}{\nu}\left(e^{-\nu t_{i-1}} N\left(z_{1} \sqrt{t_{i-1}}+\frac{z_{2}}{\sqrt{t_{i-1}}}\right)-e^{-\nu t_{i}} N\left(z_{1} \sqrt{t_{i}}+\frac{z_{2}}{\sqrt{t_{i}}}\right)\right) \\
+\frac{1}{2 \nu}\left(\frac{z_{1}}{z_{3}}+1\right) e^{z_{2}\left(z_{3}-z_{1}\right)}\left(N\left(z_{3} \sqrt{t_{i}}+\frac{z_{2}}{\sqrt{t_{i}}}\right)-N\left(z_{3} \sqrt{t_{i-1}}+\frac{z_{2}}{\sqrt{t_{i-1}}}\right)\right) \\
+\frac{1}{2 \nu}\left(\frac{z_{1}}{z_{3}}-1\right) e^{-z_{2}\left(z_{3}+z_{1}\right)}\left(N\left(z_{3} \sqrt{t_{i}}-\frac{z_{2}}{\sqrt{t_{i}}}\right)-N\left(z_{3} \sqrt{t_{i-1}}-\frac{z_{2}}{\sqrt{t_{i-1}}}\right)\right), \quad(28) \\
I_{x}\left(t_{i-1}, t_{i}, x, y, z, \phi, \nu\right)=\frac{1}{\nu}\left(\frac{e^{-\nu t_{i-1}}}{\sqrt{t_{i-1}}} n\left(z_{1} \sqrt{t_{i-1}}+\frac{z_{2}}{\sqrt{t_{i-1}}}\right)-\frac{e^{-\nu t_{i}}}{\sqrt{t_{i}}} n\left(z_{1} \sqrt{t_{i}}+\frac{z_{2}}{\sqrt{t_{i}}}\right)\right) \frac{1}{\sigma x} \\
+\frac{1}{2 \nu}\left(\frac{z_{1}+z_{3}}{z_{3}}\right)\left[e^{z_{2}\left(z_{3}-z_{1}\right)}\left(z_{3}-z_{1}\right)\left(N\left(z_{3} \sqrt{t_{i}}+\frac{z_{2}}{\sqrt{t_{i}}}\right)-N\left(z_{3} \sqrt{t_{i-1}}+\frac{z_{2}}{\sqrt{t_{i-1}}}\right)\right)\right. \\
\left.+e^{z_{2}\left(z_{3}-z_{1}\right)}\left(n\left(z_{3} \sqrt{t_{i}}+\frac{z_{2}}{\sqrt{t_{i}}}\right) \frac{1}{\sqrt{t_{i}}}-n\left(z_{3} \sqrt{t_{i-1}}+\frac{z_{2}}{\sqrt{t_{i-1}}}\right) \frac{1}{\sqrt{t_{i-1}}}\right)\right] \frac{1}{\sigma x} \\
-\frac{1}{2 \nu}\left(\frac{z_{1}-z_{3}}{z_{3}}\right)\left[e^{-z_{2}\left(z_{3}+z_{1}\right)}\left(z_{3}+z_{1}\right)\left(N\left(z_{3} \sqrt{t_{i}}-\frac{z_{2}}{\sqrt{t_{i}}}\right)-N\left(z_{3} \sqrt{t_{i-1}}-\frac{z_{2}}{\sqrt{t_{i-1}}}\right)\right)\right. \\
\left.+e^{-z_{2}\left(z_{3}+z_{1}\right)}\left(n\left(z_{3} \sqrt{t_{i}}-\frac{z_{2}}{\sqrt{t_{i}}}\right) \frac{1}{\sqrt{t_{i}}}-n\left(z_{3} \sqrt{t_{i-1}}-\frac{z_{2}}{\sqrt{t_{i-1}}}\right) \frac{1}{\sqrt{t_{i-1}}}\right)\right] \frac{1}{\sigma x},
\end{gathered}
$$

\footnotetext{
${ }^{2}$ See the Appendix for the derivation of these functions.
} 
where

$$
z_{1}=\frac{\left(r-\delta-z+\phi \sigma^{2} / 2\right)}{\sigma}, \quad z_{2}=\frac{\ln (x / y)}{\sigma} \quad \text { and } \quad z_{3}=\sqrt{z_{1}^{2}+2 \nu}
$$

To find the coefficients, we must solve the system of four equations $(27)$ for $j=1,2, \ldots, M$. At each step $j$, the above system is solved using a Newton method.

The approximation procedure of American continuous-installment puts proceeds in the same way as for calls. Let $P^{\mathrm{CI}}$ be the approximated initial premium of an American continuous-installment put corresponding to the approximated optimal exercise and stopping boundaries by $M$-piece exponential functions $F_{j} e^{f_{j} t}$ and $G_{j} e^{-g_{j} t}$, for $j=1,2, \ldots, M$, respectively. Then $P^{\mathrm{CI}}$ is given by

$$
P^{\mathrm{CI}}= \begin{cases}\left(K-S_{0}\right) & \text { if } \quad S_{0} \leq F_{M} \\ P\left(M, S_{0}, F, G, f, g, \phi, \nu, T\right) & \text { if } \quad F_{M}<S_{0}<G_{M} \\ 0 & \text { if } \quad S_{0} \geq G_{M},\end{cases}
$$

where

$$
\begin{aligned}
& P(j, x, F, G, f, g, \phi, \nu, \tau)= p(x, K, \tau)+\frac{1}{r}(q+r K)\left(1-e^{-r \tau}\right)-x\left(1-e^{-\delta \tau}\right) \\
&+\left(x \sum_{i=1}^{j} \delta I\left(t_{i-1}, t_{i}, x, F_{j-i+1} e^{f_{j-i+1}(T-\tau)}, f_{j-i+1}, 1, \delta\right)\right. \\
&\left.\quad-(q+r K) \sum_{i=1}^{j} I\left(t_{i-1}, t_{i}, x, F_{j-i+1} e^{f_{j-i+1}(T-\tau)}, f_{j-i+1},-1, r\right)\right) \\
&-\frac{q}{r}\left(1-e^{-r \tau}\right)+q \sum_{i=1}^{j} I\left(t_{i-1}, t_{i}, x, G_{j-i+1} e^{-g_{j-i+1}(T-\tau)},-g_{j-i+1},-1, r\right) .
\end{aligned}
$$

As for calls, applying the value-match and high-contact conditions (18-21) at each time step $t_{j}$, we can determine the coefficients $F_{j}, f_{j}, G_{j}$ and $g_{j}, j=1,2, \ldots, M$.

\subsection{Solving the Inhomogeneous Black-Scholes PDE with Finite Dif- ferences}

The valuation of the initial premium of an American continuous-installment option by finite differences is obtained with the Crank-Nicolson method. For the call, the inhomogeneous Black-Scholes PDE and the final and boundary conditions have been defined in (7) and (8-12). For the put, these are defined in (16) and (17-21). For discretization, a uniform 
grid in space and time is used. To achieve greater accuracy, critical points are fixed midway between two grid points in space. The optimal exercise problem is solved simply by taking the maximum between the continuation value and the option payoff. This technique is known as the explicit payout method. Other techniques consider a PSOR or a Newton method to solve the linear complementarity problem (e.g., Coleman et al. (2002)). The optimal stopping problem is solved in a similar way by taking only positive continuation values.

\subsection{Valuation with a Monte Carlo Method}

We modify the least-squares Monte Carlo method introduced by Longstaff and Schwartz (2001) to accommodate the pricing of the American continuous installment options. Let us consider a discrete-time sample path $S_{i}, i=0,1, \ldots, M$ for the price of an underlying asset, with $M=T / \Delta_{t}$, where $T$ is the time to maturity and $\Delta_{t}$ is the time discretization. For European-style options the price is given by

$$
E\left(e^{-r T} f\left(S_{M}\right)\right)
$$

where $f(\cdot)$ denotes the payoff function and $E(\cdot)$ the expectation under the risk-neutral measure.

When we consider on early exercise, the value of the contract for each simulated time instant $i$ corresponds to the maximum between the intrinsic value $f\left(S_{i}\right)$ and the expected continuation value. Therefore at time step $i$, the value $V_{i}\left(S_{i}\right)$ of the option, conditional on $S_{i}$, is

$$
V_{i}\left(S_{i}\right)=\max \left\{f\left(S_{i}\right), E_{i}\left(e^{-r \Delta_{t}} V_{i+1}\left(S_{i+1}\right) \mid S_{i}\right)\right\}
$$

where the function $V(\cdot)$ is defined recursively for $i=M-1, M-2, \ldots, 0$. The value of $V_{M}\left(S_{M}\right)$ is simply $f\left(S_{M}\right)$, i.e., the payoff at maturity. Longstaff and Schwartz (2001) approximates the conditional expectation of the continuation value $E_{i}(\cdot)$ by a linear regression of the present value of $V_{i+1}\left(S_{i+1}\right)$ at $i$ on a set of polynomials of the current asset price $S_{i}$. To get observations for the regression, we have to replicate the sample path of the underlying asset price. The $j$ th replication for the asset price is denoted by $S_{i}^{j}$, and correspondingly the $j$ th replication of the continuation value, which is the present value of $V_{i+1}^{j}\left(S_{i+1}^{j}\right)$, is 
denoted by $y_{i}^{j}$. Regressing on a second-order polynomial, the approximation of $y_{i}^{j}$ is

$$
y_{i}^{j} \cong \alpha_{1}+\alpha_{2} S_{i}^{j}+\alpha_{3}\left(S_{i}^{j}\right)^{2},
$$

and the conditional expectation of the continuation value $E_{i}\left(y_{i}^{j}\right)$ is given by $\hat{y}_{i}^{j}=\hat{\alpha}_{1}+$ $\hat{\alpha}_{2} S_{i}^{j}+\hat{\alpha}_{3}\left(S_{i}^{j}\right)^{2}$, where $\hat{\alpha}_{k}, k=1,2,3$, are the estimated regression coefficients.

In the case of continuously-paid installments at a constant rate $q$, the continuation value $y_{i}^{j}$ becomes

$$
e^{-r \Delta_{t}} V_{i+1}^{j}\left(S_{i+1}^{j}\right)-\frac{q}{r}\left(1-e^{-r \Delta_{t}}\right)
$$

and we use the same regression for the estimation of the conditional expectation. The decision for early exercise at time $i$, for a sample $j$, is taken if

$$
f\left(S_{i}^{j}\right)>\hat{y}_{i}^{j},
$$

where $j \in J_{i}^{E}$, the set of paths that are in-the-money at time $i$. The decision for early stopping is taken if

$$
\hat{y}_{i}^{j}<0 \text {, }
$$

where $j \in J_{i}^{S}$, the set of paths that are out-of-the-money at time $i$. The sets $J_{i}^{E}$ and $J_{i}^{S}$ constitute a partition of the set $J$ of replicated paths. It should be noticed that the conditional expectation $\hat{y}_{i}^{j}$ is estimated separately on the set $J_{i}^{E}$ and the set $J_{i}^{S}$.

Therefore the initial value of the option at time step $i$, conditional on $S_{i}^{j}$, is

$$
V_{i}^{j}\left(S_{i}^{j}\right)= \begin{cases}\max \left\{f\left(S_{i}^{j}\right), E_{i}\left(e^{-r \Delta_{t}} V_{i+1}^{j}\left(S_{i+1}^{j}\right) \mid S_{i}^{j}\right)\right\} & \text { if } j \in J_{i}^{E} \\ \max \left\{0, E_{i}\left(e^{-r \Delta_{t}} V_{i+1}^{j}\left(S_{i+1}^{j}\right) \mid S_{i}^{j}\right)\right\} & \text { if } j \in J_{i}^{S} .\end{cases}
$$

The computation of the option price is now achieved through the Algorithm 1, which provides a skeleton for the implementation of a computer code. 


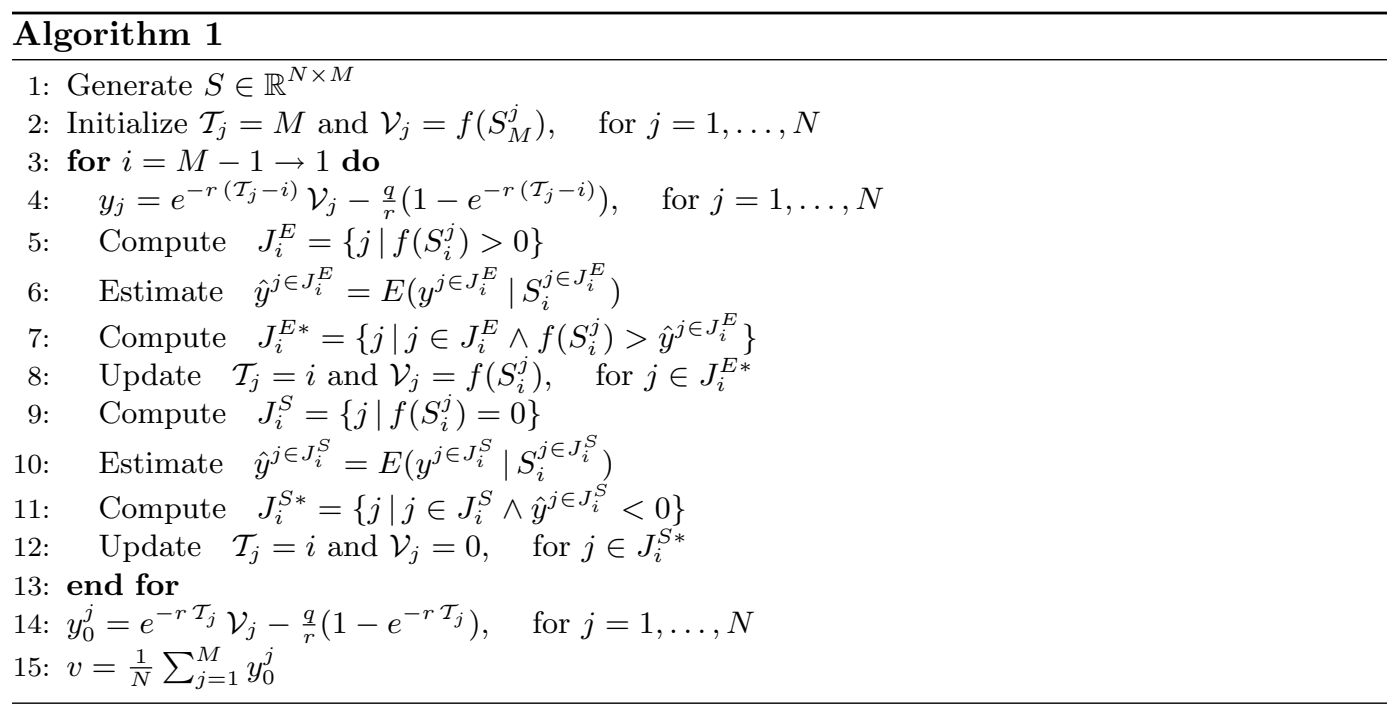

Statements 5-8 consider the case where early exercise has to be checked and statements $9-12$ where stopping has to be checked. The sets $J^{E *}$ and $J^{S *}$ correspond respectively to the paths where early exercising or stopping has taken place. Element $j$ of array $\mathcal{T}$ informs us about the time step where the early exercise or stopping decision has been taken for the $j$ th path. The intrinsic value of the option at time step $\mathcal{T}_{j}$ is given in $\mathcal{V}_{j}$. In statement 14 , the option value at time 0 for each path is saved in $y_{0}$, and, in statement 15 , the average of these values is computed.

The convergence of this method is analyzed in Glassermann and $\mathrm{Yu}$ (2004), where the choice of the order of the polynomial approximating $E(\cdot)$ is discussed in conjunction with the number $N$ of path replications and time steps $M$.

\section{Numerical Results and Discussions}

In this section we report and compare numerical results obtained with each of the three methods for several values of some relevant parameters. All algorithms have been implemented in Matlab 7.xx and the results are reported in Table 1. 


\begin{tabular}{|c|c|c|c|c|c|c|c|c|c|}
\hline & & & & FDM & & MEF & & Monte & Carlo \\
\hline$\sigma$ & $S_{0}$ & $\mathrm{~T}$ & $q$ & & $M=2$ & $M=6$ & $M=12$ & & (s.e.) \\
\hline \multirow{18}{*}{0.20} & \multirow{6}{*}{96} & \multirow{3}{*}{$3 / 12$} & 1 & 2.0700 & 2.0670 & 2.0695 & 2.0699 & 2.0702 & $(.010)$ \\
\hline & & & 3 & 1.6812 & 1.6738 & 1.6802 & 1.6812 & 1.6808 & $(.011)$ \\
\hline & & & 8 & .8945 & .8858 & .8941 & .8952 & .8927 & (.009) \\
\hline & & \multirow{3}{*}{1} & 1 & 5.2789 & 5.2669 & 5.2766 & 5.2783 & 5.2777 & $(.022)$ \\
\hline & & & 3 & 3.8362 & 3.8132 & 3.8320 & 3.8344 & 3.8361 & $(.021)$ \\
\hline & & & 8 & 1.4232 & 1.4164 & 1.4231 & 1.4239 & 1.4181 & $(.020)$ \\
\hline & \multirow{6}{*}{100} & \multirow{3}{*}{$3 / 12$} & 1 & 3.8410 & 3.8380 & 3.8405 & 3.8409 & 3.8407 & $(.010)$ \\
\hline & & & 3 & 3.4293 & 3.4211 & 3.4281 & 3.4291 & 3.4286 & $(.011)$ \\
\hline & & & 8 & 2.5477 & 2.5362 & 2.5468 & 2.5482 & 2.5455 & $(.011)$ \\
\hline & & \multirow{3}{*}{1} & 1 & 7.2717 & 7.2594 & 7.2693 & 7.2710 & 7.2712 & $(.023)$ \\
\hline & & & 3 & 5.7884 & 5.7654 & 5.7853 & 5.7878 & 5.7878 & $(.022)$ \\
\hline & & & 8 & 3.1951 & 3.1864 & 3.1946 & 3.1957 & 3.1900 & $(.016)$ \\
\hline & \multirow{6}{*}{104} & \multirow{3}{*}{$3 / 12$} & 1 & 6.2438 & 6.2411 & 6.2433 & 6.2437 & 6.2441 & $(.011)$ \\
\hline & & & 3 & 5.8427 & 5.3500 & 5.8415 & 5.8424 & 5.8423 & (.009) \\
\hline & & & 8 & 5.0192 & 5.0095 & 5.0185 & 5.0198 & 5.0175 & $(.008)$ \\
\hline & & \multirow{3}{*}{1} & 1 & 9.5839 & 9.5718 & 9.5816 & 9.5833 & 9.5898 & $(.025)$ \\
\hline & & & 3 & 8.1123 & 8.0883 & 8.1079 & 8.1104 & 8.1122 & $(.016)$ \\
\hline & & & 8 & 5.5935 & 5.5859 & 5.5933 & 5.5942 & 5.5851 & $(.015)$ \\
\hline \multirow{18}{*}{0.30} & \multirow{6}{*}{96} & \multirow{3}{*}{$3 / 12$} & 1 & 3.9032 & 3.8996 & 3.9026 & 3.9031 & 3.9051 & $(.019)$ \\
\hline & & & 3 & 3.4926 & 3.4831 & 3.4910 & 3.4922 & 3.4928 & $(.016)$ \\
\hline & & & 8 & 2.5826 & 2.5668 & 2.5805 & 2.5823 & 2.5825 & $(.019)$ \\
\hline & & \multirow{3}{*}{1} & 1 & 8.9756 & 8.9607 & 8.9732 & 8.9755 & 8.9753 & $(.043)$ \\
\hline & & & 3 & 7.4528 & 7.4233 & 7.4484 & 7.4515 & 7.4549 & $(.036)$ \\
\hline & & & 8 & 4.4203 & 4.3908 & 4.4161 & 4.4196 & 4.4200 & $(.036)$ \\
\hline & \multirow{6}{*}{100} & \multirow{3}{*}{$3 / 12$} & 1 & 5.8118 & 5.8081 & 5.8111 & 5.8116 & 5.8138 & $(.020)$ \\
\hline & & & 3 & 5.3909 & 5.3810 & 5.3892 & 5.3905 & 5.3909 & $(.018)$ \\
\hline & & & 8 & 4.4420 & 4.4248 & 4.4396 & 4.4416 & 4.4418 & $(.015)$ \\
\hline & & \multirow{3}{*}{1} & 1 & 11.0836 & 11.0682 & 11.0810 & 11.0834 & 11.0841 & $(.045)$ \\
\hline & & & 3 & 9.5415 & 9.5109 & 9.5369 & 9.5403 & 9.5443 & (.038) \\
\hline & & & 8 & 6.4218 & 6.3903 & 6.4173 & 6.4211 & 6.4208 & $(.030)$ \\
\hline & \multirow{6}{*}{104} & \multirow{3}{*}{$3 / 12$} & 1 & 8.1425 & 8.1388 & 8.1417 & 8.1422 & 8.1426 & $(.023)$ \\
\hline & & & 3 & 7.7246 & 7.7148 & 7.7229 & 7.7241 & 7.7236 & $(.016)$ \\
\hline & & & 8 & 6.7900 & 6.7733 & 6.7877 & 6.7895 & 6.7910 & $(.013)$ \\
\hline & & \multirow{3}{*}{1} & 1 & 13.4023 & 13.3863 & 13.3993 & 13.4017 & 13.4059 & $(.036)$ \\
\hline & & & 3 & 11.8595 & 11.8286 & 11.8548 & 11.8585 & 11.8582 & $(.029)$ \\
\hline & & & 8 & 8.7391 & 8.7076 & 8.7346 & 8.7384 & 8.7348 & $(.023)$ \\
\hline
\end{tabular}

Table 1: Initial premiums of American continuous-installment calls $(K=100$ and $\delta=0.04)$.

For the finite-difference method, we use 600 steps between 0 and 200 for the asset price and 400 time steps per quarter of a year. The multipiece exponential function (MEF) method has been tested for $M=2, M=6$ and $M=12$. The results for the Monte Carlo method are based on 100000 antithetic paths and a fourth-order Hermite polynomial for the regressions. The number of time steps used for this method is 80 per quarter of 
a year. Following Glassermann and $\mathrm{Yu}$ (2004, p. 18) these settings satisfy the conditions for convergence. To estimate the standard errors, we compute a statistic with 50 initial premiums. The values reported in the table are the medians of this statistic.

Comparing the results obtained by the MEF method for $M=12$ with the results given by the other two methods we see, in Table 1, that the approximations coincide from two to five digits. If the MEF method is used with $M=2$, we get from one to three correct digits.

In terms of computational efficiency, the finite-difference method result to be fastest with a computational time of less than 1 second to calculate the initial premiums at all grid points for a 3-month American continuous-installment call. The optimal stopping and exercise boundaries can be derived from the values on the space-time grid.

The MEF method with $M=12$ needs roughly 10 seconds to solve the pricing problem for the same option and provides the initial premium for a single value of $S_{t}$, as well as a pointwise approximation of the boundaries. If we consider $M=2$, the computational time becomes comparable to that of the finite differences. A interesting feature of this method is the determination of the three components in which the initial premium has been decomposed via integral representation. A difficulty of the MEF method may consist in the appropriate choice of the initial values when one solving the non-linear system (27). The Monte Carlo approach needs approximatively 14 seconds to find the initial premium. Since the result is of random nature we need to compute confidence intervals which imply repeated evaluations of the initial premium. An advantage of the Monte Carlo method is that it can be extended easily to exotic payoffs and multifactor option.

The left panel in Figure 1 presents the initial premium function $C\left(S_{t}, t ; q\right)$ and the optimal stopping and exercise boundaries, both calculated by finite differences. The right panel in Figure 1 shows how each method approximates the boundaries. The approximations of the exercise and stopping boundaries obtained by the finite-difference method are respectively the solid and the dotted lines. The crosses and circles represent the twelve-piece exponential exercise and stopping boundaries, respectively. The clouds of points along the boundaries are the optimal stopping and exercise decisions for each path in the Monte Carlo method. 

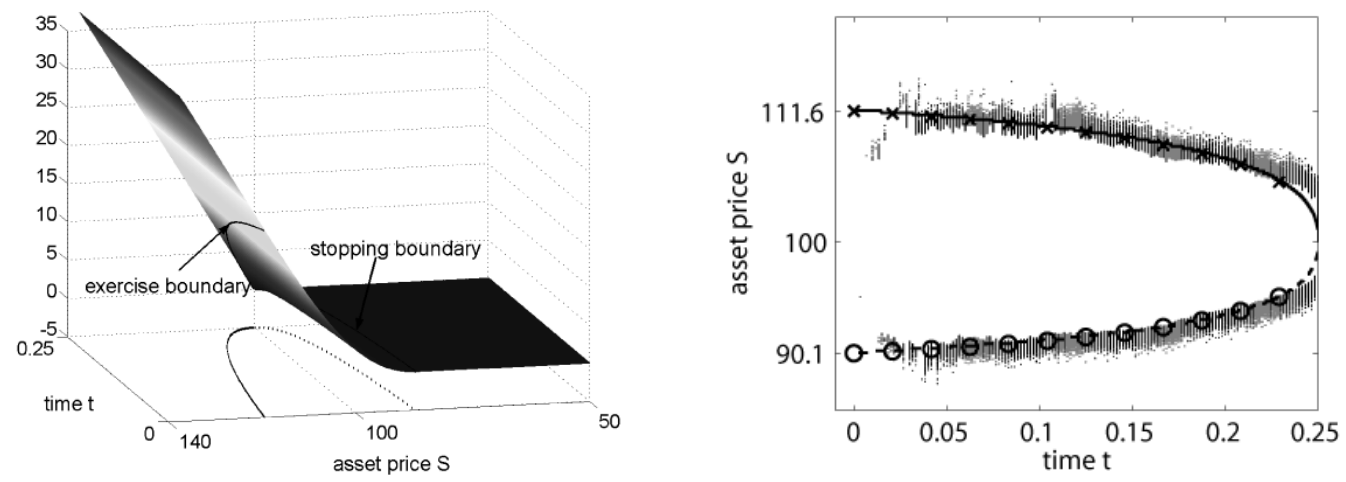

Figure 1: Left panel: Initial premium function $C\left(S_{t}, t ; q\right)$ of an American continuousinstallment call $(K=100, T=3 / 12, \sigma=0.2, r=0.05, \delta=0.04$ and $q=8)$. Right panel: Optimal stopping and exercise boundaries approximated by finite differences, the twelve-piece exponential boundaries and the stopping and exercise decisions of the Monte Carlo simulations.

\section{Concluding Remarks}

We have presented three alternative approaches for solving the free boundary-value problem of American continuous-installment options. First we derived the inhomogeneous BlackScholes PDE for continuous-installment options using a combination of hedging and riskneutral valuation arguments. This result allows the derivation of an integral representation for the initial premium of these options, using the results in Carr et al. (1992), Jacka (1991) and $\operatorname{Kim}(1990)$.

The multipiece exponential function (MEF) method allows an approximation in closed form to the valuation formulas for the American continuous-installment options. To test the MEF method we adapted two existing numerical methods to the pricing problem of the nonstandard American options. All three methods produce similar results from which we conclude the soundness of our approaches.

The focus of this paper is on American continuous-installment calls. However, by presenting a mathematically and computationally meaningful way to analyze the premature stopping of American options, this study enhances applications of the contingent-claims approach to investment problems in general. For example, investments involving periodic payments that can be stopped at any time can be analyzed using the framework developed in this paper. 


\section{Appendix}

\section{Derivation of functions $I(\cdot)$ and $I_{x}(\cdot)$}

Let us assume that for the generic interval $\left[t_{i-1}, t_{i}\right]$ the stopping and exercise boundaries $A_{t}$ and $B_{t}$ are approximated by exponential functions $A e^{a t}$ and $B e^{-b t}$, respectively. To make use of this approximation, the integrals in equations (13) and (23) can be evaluated in closed form for this interval. We first consider the integral

$$
I_{1}=\int_{t_{i-1}}^{t_{i}} e^{-r t} N\left(d_{2}\left(S_{0}, A e^{a t}, t\right)\right) d t .
$$

Defining $x_{1}=\left(r-\delta-a-\sigma^{2} / 2\right) / \sigma, x_{2}=\ln \left(S_{0} / A\right) / \sigma$, we have that $d_{2}\left(S_{0}, A_{t}, t\right)=x_{1} t^{1 / 2}+$ $x_{2} t^{-1 / 2}$. Integration by parts yields

$$
\begin{aligned}
I_{1}= & \frac{1}{r}\left(e^{-r t_{i-1}} N\left(x_{1} t_{i-1}^{1 / 2}+x_{2} t_{i-1}^{-1 / 2}\right)-e^{-r t_{i}} N\left(x_{1} t_{i}^{1 / 2}+x_{2} t_{i}^{-1 / 2}\right)\right) \\
& +\frac{e^{-x_{1} x_{2}}}{r \sqrt{2 \pi}} \int_{t_{i-1}}^{t_{i}} e^{-\frac{1}{2}\left(x_{3}^{2} t+x_{2}^{2} t^{-1}\right)}\left(\frac{x_{1}}{2} t^{-1 / 2}-\frac{x_{2}}{2} t^{-3 / 2}\right) d t,
\end{aligned}
$$

where $x_{3}=\sqrt{x_{1}^{2}+2 r}$. By making use of the following identities

$$
\begin{aligned}
& d N\left(x_{3} t^{1 / 2}+x_{2} t^{-1 / 2}\right)=\frac{e^{-x_{3} x_{2}}}{\sqrt{2 \pi}} e^{-\frac{1}{2}\left(x_{3}^{2} t+x_{2}^{2} t^{-1}\right)}\left(\frac{x_{3}}{2} t^{-1 / 2}-\frac{x_{2}}{2} t^{-3 / 2}\right) \\
& d N\left(x_{3} t^{1 / 2}-x_{2} t^{-1 / 2}\right)=\frac{e^{x_{3} x_{2}}}{\sqrt{2 \pi}} e^{-\frac{1}{2}\left(x_{3}^{2} t+x_{2}^{2} t^{-1}\right)}\left(\frac{x_{3}}{2} t^{-1 / 2}+\frac{x_{2}}{2} t^{-3 / 2}\right),
\end{aligned}
$$

we get

$$
\begin{aligned}
I_{1}= & \frac{1}{r}\left(e^{-r t_{i-1}} N\left(x_{1} \sqrt{t_{i-1}}+\frac{x_{2}}{\sqrt{t_{i-1}}}\right)-e^{-r t_{i}} N\left(x_{1} \sqrt{t_{i}}+\frac{x_{2}}{\sqrt{t_{i}}}\right)\right) \\
& +\frac{1}{2 r}\left(\frac{x_{1}}{x_{3}}+1\right) e^{x_{2}\left(x_{3}-x_{1}\right)}\left(N\left(x_{3} \sqrt{t_{i}}+\frac{x_{2}}{\sqrt{t_{i}}}\right)-N\left(x_{3} \sqrt{t_{i-1}}+\frac{x_{2}}{\sqrt{t_{i-1}}}\right)\right) \\
& +\frac{1}{2 r}\left(\frac{x_{1}}{x_{3}}-1\right) e^{-x_{2}\left(x_{3}+x_{1}\right)}\left(N\left(x_{3} \sqrt{t_{i}}-\frac{x_{2}}{\sqrt{t_{i}}}\right)-N\left(x_{3} \sqrt{t_{i-1}}-\frac{x_{2}}{\sqrt{t_{i-1}}}\right)\right) .
\end{aligned}
$$

From the above equation follows immediately that

$$
\begin{aligned}
I_{2}= & \int_{t_{i-1}}^{t_{i}} e^{-r t} N\left(d_{2}\left(S_{0}, B e^{-b t}, t\right)\right) d t \\
= & \frac{1}{r}\left(e^{-r t_{i-1}} N\left(x_{4} \sqrt{t_{i-1}}+\frac{x_{5}}{\sqrt{t_{i-1}}}\right)-e^{-r t_{i}} N\left(x_{4} \sqrt{t_{i}}+\frac{x_{5}}{\sqrt{t_{i}}}\right)\right) \\
& +\frac{1}{2 r}\left(\frac{x_{4}}{x_{6}}+1\right) e^{x_{5}\left(x_{6}-x_{4}\right)}\left(N\left(x_{6} \sqrt{t_{i}}+\frac{x_{5}}{\sqrt{t_{i}}}\right)-N\left(x_{6} \sqrt{t_{i-1}}+\frac{x_{5}}{\sqrt{t_{i-1}}}\right)\right) \\
& +\frac{1}{2 r}\left(\frac{x_{4}}{x_{6}}-1\right) e^{-x_{5}\left(x_{6}+x_{4}\right)}\left(N\left(x_{6} \sqrt{t_{i}}-\frac{x_{5}}{\sqrt{t_{i}}}\right)-N\left(x_{6} \sqrt{t_{i-1}}-\frac{x_{5}}{\sqrt{t_{i-1}}}\right)\right),
\end{aligned}
$$


where $x_{4}=\left(r-\delta+b-\sigma^{2} / 2\right) / \sigma, x_{5}=\ln \left(S_{0} / B\right) / \sigma$, and $x_{6}=\sqrt{x_{4}^{2}+2 r}$.

If we define $y_{1}=\left(r-\delta+b+\sigma^{2} / 2\right) / \sigma, y_{2}=\ln \left(S_{0} / B\right) / \sigma, y_{3}=\sqrt{y_{1}^{2}+2 \delta}$, a similar derivation would yield

$$
\begin{aligned}
I_{3}= & \int_{t_{i-1}}^{t_{i}} e^{-\delta t} N\left(d_{1}\left(S_{0}, B e^{-b t}, t\right)\right) d t \\
= & \frac{1}{\delta}\left(e^{-\delta t_{i-1}} N\left(y_{1} \sqrt{t_{i-1}}+\frac{y_{2}}{\sqrt{t_{i-1}}}\right)-e^{-\delta t_{i}} N\left(y_{1} \sqrt{t_{i}}+\frac{y_{2}}{\sqrt{t_{i}}}\right)\right) \\
& +\frac{1}{2 \delta}\left(\frac{y_{1}}{y_{3}}+1\right) e^{y_{2}\left(y_{3}-y_{1}\right)}\left(N\left(y_{3} \sqrt{t_{i}}+\frac{y_{2}}{\sqrt{t_{i}}}\right)-N\left(y_{3} \sqrt{t_{i-1}}+\frac{y_{2}}{\sqrt{t_{i-1}}}\right)\right) \\
& +\frac{1}{2 \delta}\left(\frac{y_{1}}{y_{3}}-1\right) e^{-y_{2}\left(y_{3}+y_{1}\right)}\left(N\left(y_{3} \sqrt{t_{i}}-\frac{y_{2}}{\sqrt{t_{i}}}\right)-N\left(y_{3} \sqrt{t_{i-1}}-\frac{y_{2}}{\sqrt{t_{i-1}}}\right)\right) .
\end{aligned}
$$

Using equation (28), the integrals $I_{1}, I_{2}$ and $I_{3}$ can be expressed uniquely as

$$
\begin{aligned}
& I_{1}=I\left(t_{i}, t_{i-1}, x, A, a,-1, r\right), \\
& I_{2}=I\left(t_{i-1}, t_{i}, x, B,-b,-1, r\right), \\
& I_{3}=I\left(t_{i-1}, t_{i}, x, B,-b, 1, \delta\right) .
\end{aligned}
$$

The function $I_{x}(\cdot)$ is the first partial derivative of (28) with respect to $x$.

\section{References}

Ben-Ameur, H., M. Breton and P. François (2004). A Dynamic Programming Approach to Price Installment Options. To appear in European Journal of Operational Research.

Carr, P., R. Jarrow and R. Myneni (1992). Alternative Characterizations of American Put Options. Mathematical Finance 2(2), 87-106.

Coleman, T.F., Y. Li and A. Verma (2002). A Newton Method for American Option Pricing. Journal of Computational Finance 5(3), 51-78.

Davis, M., W. Schachermayer and R. Tompkins (2001). Pricing, No-Arbitrage Bounds and Robust Hedging of Instalment Options. Quantitative Finance 1(6), 597-610.

Davis, M., W. Schachermayer and R. Tompkins (2002). Installment Options and Static Hedging. Journal of Risk Finance 3(2), 46-52.

Davis, M., W. Schachermayer and R. Tompkins (2003). The Evaluation of Venture Capital As an Instalment Option: Valuing Real Options Using Real Options. To appear in Zeitschrift für Betriebswirtschaft. 
Geske, R. (1977). The Valuation of Corporate Liabilities as Compound Options. Journal of Financial and Quantitative Analysis 12(4), 541-552.

Glassermann, P. and B. Yu (2004). Number of Paths Versus Number of Basis Functions in American Option Pricing. To appear in The Annals of Applied Probability.

Jacka, S.D. (1991). Optimal Stopping and the American Put. Mathematical Finance 1(2), 114.

Ju, N. (1998). Pricing an American Option by Approximating Its Early Exercise Boundary as a Multpiece Expontential Function. Review of Financial Studies 11(3), 627-646.

Kim, I.J. (1990). The Analytical Valuation of American Options. Review of Financial Studies 3(4), 547-572.

Kolodner, I.I. (1956). Free Boundary Problem for the Heat Equation with Applications to Problems of Change of Phase. Communications in Pure and Applied Mathematics 9, 1-31.

Longstaff, F.A. and E.S. Schwartz (2001). Valuing American Options by Simuation: A Simple Least-Square Approach. Review of Financial Studies 14(1), 113-147.

McKean, H.P. (1965). Appendix: A Free Boundary Problem for the Heat Equation Arising from a Problem in Mathematical Economics. Industrial Management Review 6, 32-39.

Selby, M.J.P. and S.D. Hodges (1987). On the Evaluation of Compound Options. Management Science 33, 347-355.

Wystup, U., S. Griebsch and C. Kühn (2004). FX Instalment Options. Working Paper.

Ziogas, A, C. Chiarella and A. Kucera (2004). A Survey of the Integral Representation of American Option Prices. Working Paper, University of Techology, Sidney. 